\title{
The Notch signaling pathway in head and neck squamous cell carcinoma: A meta-analysis
}

\author{
Yu-Yue Zhao ${ }^{A-F}$, Guang-Tao Yu ${ }^{A-D}$, Ting Xiao ${ }^{B, C}$, Jian Hu ${ }^{A, D-F}$ \\ The State Key Laboratory Breeding Base of Basic Science of Stomatology (Hubei-MOST) and Key Laboratory for Oral Biomedical Ministry of Education, \\ School and Hospital of Stomatology, Wuhan University, China
}

A - research concept and design; B - collection and/or assembly of data; $\mathrm{C}$ - data analysis and interpretation; $D$ - writing the article; $E$ - critical revision of the article; $F$ - final approval of article

\section{Address for correspondence \\ Jian Hu}

E-mail:00008460@whu.edu.cn

Funding sources

None declared

\section{Conflict of interest}

None declared

\section{Acknowledgements}

We would like to thank Daniel Ford for language editing of this paper. We apologize colleagues whose primary research papers may not have been cited due to space contraints

Received on July 29, 2015

Revised on May 04, 2016

Accepted on July 04, 2016

\section{Abstract}

Background. The Notch signaling pathway has been associated with the regulation of self-renewal capacity, cell cycle exit, and survival. However, the relationship between the Notch signaling pathway and HNSCC remains controversial.

Objectives. A meta-analysis was conducted to evaluate the role of Notch signaling pathway in HNSCC

Material and methods. Relevant studies published until March 31, 2015 were identified by searching the PubMed, EMBASE and Ovid database.

Results. A total of 9 articles were eligible for this meta-analysis. The meta-analysis results showed that the expression of Notch1, Notch3 and NICD was significantly higher in HNSCC as compared with control tissue. There was no significant difference in Jagged1 and HES1 expression between HNSCC and control tissue. Stratified analysis results showed that the expression of Notch1 was significantly higher in poor differentiation, III and IV stage and positive lymph node metastasis patients. Additionally, over-expression of Notch1, NICD, HES1 and DLL4 significantly predicted poor OS in HNSCC patients.

Conclusions. The Notch signaling pathway plays an important role in tumor development of HNSCC. Inhibition of the Notch signaling pathway is a potential therapeutic method of HNSCC.

Key words: prognosis, Notch, head and neck squamous cell carcinoma, systematic review and metaanalysis

DOI

10.17219/acem/64000

Copyright

Copyright by Author(s)

This is an article distributed under the terms of the

Creative Commons Attribution Non-Commercial License

(http://creativecommons.org/licenses/by-nc-nd/4.0/) 
Head and neck squamous cell carcinoma (HNSCC) is one of the most frequent cancers with significant morbidity and mortality around the world. Despite the advancements in surgery and radiochemothreapy, the 5 -year survival rate of patients with advanced HNSCC has only marginally improved. ${ }^{1}$ Therefore, it is urgent to identify the biomarkers to screen out high risk patients and evaluate the prognosis of HNSCC.

The Notch signaling pathway has been associated with the regulation of self-renewal capacity, cell cycle exit, and survival. ${ }^{2}$ In mammals, this signaling is comprised of 4 receptor isoform (Notch1, Notch2, Notch3, Notch4) and 5 ligands: Delta-like 1 (DLL1), Delta-like 3 (DLL3), Deltalike 4 (DLL4), Jagged1, Jagged2. ${ }^{3}$ The pathway is activated when one cell expressing the appropriate ligand interacts with another cell expressing a Notch receptor. Next, the transmembrane Notch receptor is subsequently cleaved by a disintegrin and metalloprotease (ADAM) metalloprotease and $\gamma$-secretase complex. Then, the cleaved product, Notch intracellular domain (NICD), translates into the nucleus to regulate the expression of target genes. ${ }^{4,5}$ The hairy/enhancer of split (HES) and hairy/enhancer of split related with YRPW motif (HEY) families are the most prominent targets proteins of the Notch pathway. ${ }^{6}$

Mechanism studies demonstrated that dysfunctional Notch signaling can lead to numerous diseases, such as breast cancer, T-cell leukemia, diabetic nephropathy. ${ }^{7-9}$ In an osteogenic sarcoma mouse model, the activation of Notch signaling was verified as a common triggering mechanism in mesenchymal cells original carcinoma. ${ }^{10}$ Nevertheless, in a tissue-specific inducible gene-target mice model, Notch1 deficiency resulted in a skin tumor by increasing and sustaining the expression of GLI family zinc finger protein 2 (Gli2). ${ }^{11}$ In HNSCC, the Notch signaling pathway has been implicated in many facets of cancer biology, including angiogenesis, cancer stem cell and drug resistance. ${ }^{12-14}$ A recent exome sequence demonstrated that Notch 1 mutations occur in approximately $15 \%$ of HNSCC. ${ }^{15}$ Moreover, Notch 1 mutations indicate a loss of function mutation and they play a tumor suppressive role through reduced target gene expression (HES1 and HEY1). ${ }^{16}$

The dual biological effect of Notch signaling pathway playing either a cancer-promoting role or a tumorsuppressing role has been debated. Therefore, we performed a meta-analysis to assess the association of the Notch signaling pathway with HNSCC and the predicted role of Notch signaling pathway in HNSCC patients.

\section{Material and methods}

\section{Search strategy}

We searched the electronic databases PubMed, EMBASE and Ovid by using the search terms: (head and neck squamous cell carcinoma or HNSCC or oral cancer or laryngeal cancer or pharyngeal cancer or tongue cancer or oropharyngeal cancer) and (notch or NICD or notch intracellular or DLL or delta or delta like or jagged or HES1 or HEY1 or notch mutation). The search was limited to English language papers. The search was updated until March 31, 2015.

\section{Inclusion and exclusion criteria}

The papers were included in the meta-analysis if they met the following criteria: (1) studies containing patient case of head and neck squamous carcinoma (HNSCC), including: oral cancer, tongue cancer, larynx cancer, oropharynx cancer and nasopharyngeal carcinoma; (2) studies measuring the expression of Notch signaling pathway proteins; (3) studies containing sufficient data to estimate the odds ratio (OR) or hazard ratio (HR) of overall survival (OS) or disease-free survival (DFS); (4) studies published as peer-reviewed and original research; (5) if there were more than one study based on a similar population, the largest or the most recent study was included. Meanwhile, studies were excluded according to any of the following criteria: (1) the study was published as a review or letters; (2) the study lacked sufficient information to calculate the OR or HR. If patient specimens were involved in different studies, the most recently published reports were included in this study.

\section{Data extraction}

The studies were independently searched and assessed by 2 reviewers (Zhao YY and Yu GT), and the inclusion of a study was decided by consensus. The following items were extracted: first author, publication year, patient distribution, specimen size, assessment method of Notch signaling pathway, the expression of Notch signaling pathway, OS and/or DFS and follow-up period.

\section{Statistical analysis}

Data statistical analysis was performed using STATA (v. 12.0, STATA Corporation, College Station). OR and 95\%CI were used to assess the relationship between the Notch signaling pathway and HNSCC, including gender, clinical stage, tumor differentiation and lymph node metastasis status. The HR was used to assess the association between the Notch signaling pathway and OS or DFS. The Q test and p-value was used to evaluate the heterogeneity. When $\mathrm{p}<0.1$ or $\mathrm{I}^{2}>50 \%$, a random effect model was applied. Otherwise, the fixed effect model was used. The sensitive analysis was applied to measure whether the heterogeneity of these studies is from one single study. Publication bias was assessed by Begg's rank correlation method. The value of $\mathrm{p}<0.05$ was considered statistically significant. 
Table 1. Characteristics of all eligible studies

\begin{tabular}{|c|c|c|c|c|c|c|c|}
\hline Ref. & Country & Year & Technique & Detected markers & $\begin{array}{c}\text { No. of } \\
\text { patients }\end{array}$ & $\begin{array}{l}\text { No. of } \\
\text { control }\end{array}$ & $\begin{array}{c}\text { Follow-up } \\
\text { (month) }\end{array}$ \\
\hline Gokulan et al. ${ }^{17}$ & India & 2014 & $\mathrm{IHC}$ & NICD/HES1 & 44 & 8 & $14-50$ \\
\hline Sun et al. ${ }^{16}$ & USA & 2014 & $\mathrm{IHC}$ & Notch1/HES1/HEY1 & 56 & 11 & na \\
\hline Yoshida et al. ${ }^{18}$ & Japan & 2013 & $\mathrm{HC}$ & Notch1/NICD/Jagged1 & 54 & 12 & na \\
\hline Zhang et al. ${ }^{19}$ & China & 2012 & $\mathrm{HC}$ & DLL4 & 311 & na & $1-120$ \\
\hline Gokulan et al. ${ }^{20}$ & India & 2011 & $\mathrm{HC}$ & Notch1 & 62 & na & $9-36$ \\
\hline Zhang et al. ${ }^{21}$ & China & 2010 & $\mathrm{IHC}$ & Notch1/Notch3/Jagged11/Jagged2 & 74 & 74 & na \\
\hline Lin et al. ${ }^{22}$ & China & 2010 & $\mathrm{HC}$ & Notch1/Jagged1 & 59 & na & 60 \\
\hline Joo et al. ${ }^{23}$ & Korea & 2008 & $\mathrm{HC}$ & Notch1/Notch3 & 51 & 5 & na \\
\hline Zhang et al. ${ }^{24}$ & China & 2008 & $\mathrm{HC}$ & Notch1 & 25 & 25 & na \\
\hline
\end{tabular}

Table 2. Subgroup analysis of the Notch1 expression of HNSCC

\begin{tabular}{|c|c|c|c|c|c|}
\hline Stratification of HNSCC & $\begin{array}{l}\text { No. of } \\
\text { patients }\end{array}$ & OR $(95 \% \mathrm{Cl})$ & $\begin{array}{l}\text { Statistical } \\
\text { method }\end{array}$ & p-value & No. of Ref \\
\hline Gender: male vs female & 128 & $1.069(0.517-2.209)$ & fixed & 0.858 & 2 \\
\hline Clinical stage: I-II vs III-IV & 128 & $0.174(0.069-0.439)$ & fixed & 0.000 & 2 \\
\hline $\begin{array}{l}\text { Lymph node: mestastasis: } \\
\text { positive vs negative }\end{array}$ & 125 & 4.205(1.767-10.007) & fixed & 0.001 & 2 \\
\hline Differentation: well vs moderate & 112 & $1.102(0.482-2.519)$ & fixed & 0.819 & 2 \\
\hline
\end{tabular}

\section{Results}

\section{Description of study}

The literature search retrieved 448 related references. After browsing the title and abstract, 38 potential studies were selected. Further, 9 studies were eligible to be included in this meta-analysis after the full text articles had been read against the inclusion and exclusion criteria (Fig. 1). ${ }^{16-24}$ An immunohistochemical (IHC) method was used to assess the Notch signaling in HNSCC. These studies were carried out in India, America, Japan, Korea and China. Among these studies, 5 focused on acceptor Notch1, 2 focused on acceptor Notch3, 2 focused on NICD, 2 focused on ligand Jagged 1 and 2 focused on target protein HES1. Only 1 study focused on Jagged 2 and HEY1, respectively. The detailed characteristics of these studies were shown in Table 1.

\section{Meta-analysis of Notch signaling in HNSCC}

Notch1: Five studies evaluated the expression of Notch1 in HNSCC and in control tissue. The combined results showed that Notch1 expression was significantly higher in HNSCC than in control tissue $(\mathrm{OR}=10.17,95 \% \mathrm{CI}$ : 2.31-44.85; a random effects analysis, $\mathrm{p}=0.002$ ) (Fig. $2 \mathrm{~A}$ ). Given the $\mathrm{I}^{2}=69.4 \%$ and $\mathrm{p}=0.011$, we next assessed the heterogeneity of these 5 studies by sensitive analysis (Fig. 2B). We found that the article of Zhang et al. was the main source of heterogeneity. Further, the combined results based on the remaining 4 studies also demonstrated that Notch1 had a significantly high expression in HNSCC as compared with control tissue $(\mathrm{OR}=16.86$, 95\% CI: 6.27-45.33; a fixed effects analysis, $\mathrm{p}=0.000$ ) (Fig. 2C). Moreover, there was no obvious heterogeneity in these 4 studies $\left(\mathrm{I}^{2}=47.7 \%, \mathrm{p}=0.125\right)$. This data indicated that Notch1 was up-regulated in HNSCC.

As shown in Table 2, we analyzed the relationship of Notch1 expression with clinical features. We found that there was no difference in Notch1 expression between males and females (OR $=1.069,95 \% \mathrm{CI}: 0.517-2.209)$. Similarly, there was also no difference in Notch 1 expression between well differentiation and poor differentiation $(\mathrm{OR}=1.102$, 95\%CI: 0.482-2.519). When stratifying for clinical stage, we found that Notch1 expression was significantly increased in III and IV stage patients as compared with I and II stage patients $(\mathrm{OR}=0.174,95 \% \mathrm{CI}$ : 0.069-0.439). And when patients presented lymph node 
Fig. 1. Flow chart of articles selection

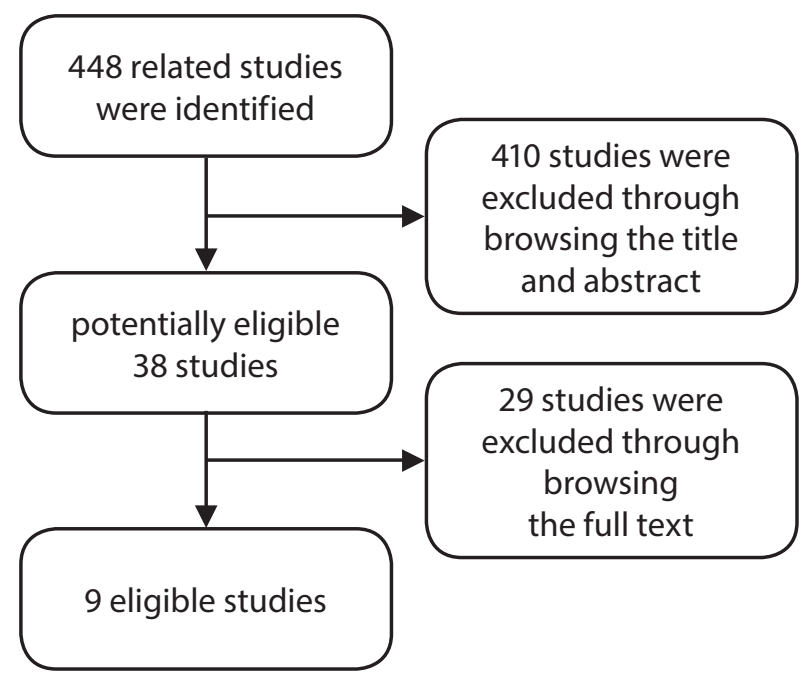

Fig. 2. (A) Forest plot for the association between Notch1 expression and HNSCC. (B) Sensitive analysis for the assessment of heterogeneity of included studies. (C) Once again forest plot for the association between Notch1 expression and HNSCC by excluding a heterogeneous study

A

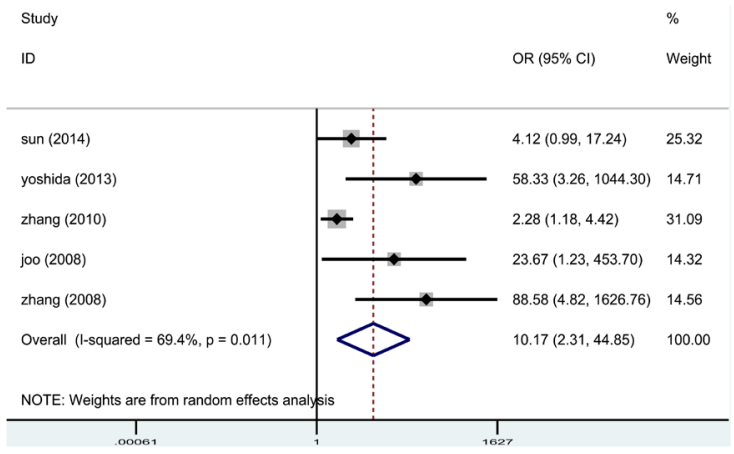

B

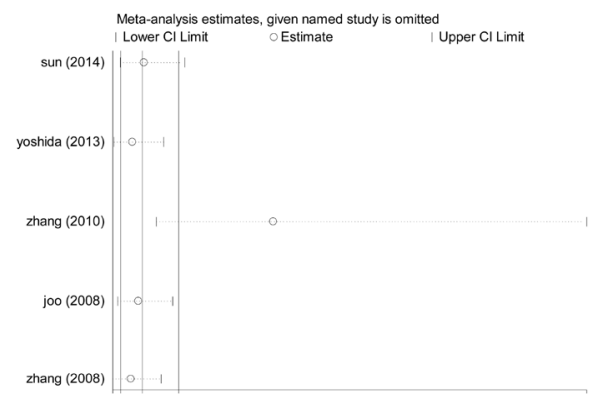

C

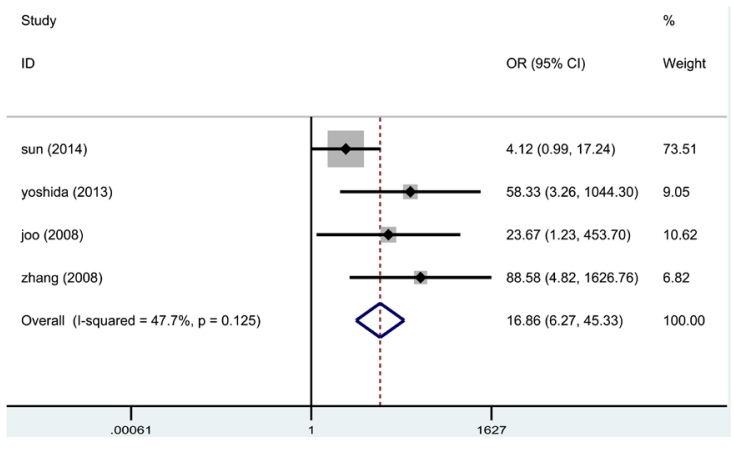

metastasis, the expression of Notch1 was also significantly up-regulated (OR = 4.205, 95\%CI: 1.767-10.007).

Notch3: The combined results based on 2 independent studies showed that Notch3 has a significantly higher expression in HNSCC than in control tissue. The OR was 3.19 (95\%CI: $1.73-5.89, \mathrm{p}=0.000)$, without any heterogeneity between studies $\left(\mathrm{I}^{2}=0.00 \%, \mathrm{p}=0.458\right.$, a fixed effects analysis) (Fig. 3A). When stratifying for age, gender, lymph node metastasis and histology, no statistically significant difference was found in HNSCC patients. When stratifying clinical stage, we found that Notch3 expression was higher in III and IV stage patient as compared with I and II stage patients. ${ }^{21}$

NICD: The combined results based on 2 independent studies showed that NICD has significantly higher expression in HNSCC than in control tissue. The OR was 57.81 (95\% CI: 6.45-518.38, $\mathrm{p}=0.000)$, without any heterogeneity between studies $\left(\mathrm{I}^{2}=0.00 \%, \mathrm{p}=0.653\right.$, a fixed effects analysis) (Fig. 3B). When stratifying for age, gender and histology, no statistically significant differences were found in HNSCC patients. When stratifying clinical stage and lymph node metastasis, NICD has significantly higher expression in III + IV stage patients and positive lymph node metastasis patients. ${ }^{17}$

Jagged1: The combined results based on 2 independent studies showed that there was no statistically significant difference in Jagged1 expression between HNSCC and control tissue. The OR was 34.79 (95\%CI: 0.23-5359.08, $\mathrm{p}=0.167)$. But the heterogeneity was detected between studies $\left(\mathrm{I}^{2}=82 \%, \mathrm{p}=0.018\right.$, a random effects analysis) (Fig. 3C). When stratifying for age, gender, clinical stage and histology, no statistically significant differences were found in HNSCC patients. When stratifying for lymph node metastasis, NICD has significantly higher expression in positive lymph node metastasis patients than in the case of negative lymph node metastasis. ${ }^{21}$

HES1: The combined results based on 2 independent studies showed that there was no statistically significant difference in HES1 expression between HNSCC and control tissue. The OR was 7.27 (95\%CI: 0.42-124.52, $\mathrm{p}=0.171)$. However, heterogeneity was detected between studies $\left(\mathrm{I}^{2}=68.5 \%, \mathrm{p}=0.075\right.$, a random effects analysis) (Fig. 3D). When stratifying for age, gender and histology, no statistically significant differences were found in $\mathrm{HN}$ SCC patients. When stratifying clinical stage and lymph node metastasis, HES1 has a significantly higher expression in III + IV stage patients and positive lymph node metastasis patients. ${ }^{17}$

Other components of Notch signaling pathway: The expression of Jagged2 was not statistically different in HNSCC and control tissue ( $\mathrm{p}=0.157)$. When stratifying clinical stage, Jagged2 has a significantly higher expression in III + IV stage patients as compared with I + II stage patients. ${ }^{21}$ The expression of HEY1 was also not statistically significant different in HNSCC and control tissue $(\mathrm{p}=0.11) .{ }^{16}$ 
Fig. 3. (A) Forest plot for the association between Notch3 and HNSCC. (B) Forest plot for the association between NICD and HNSCC. (C) Forest plot for the association between Jagged1 and HNSCC. (D) Forest plot for the association between HES1 and HNSCC
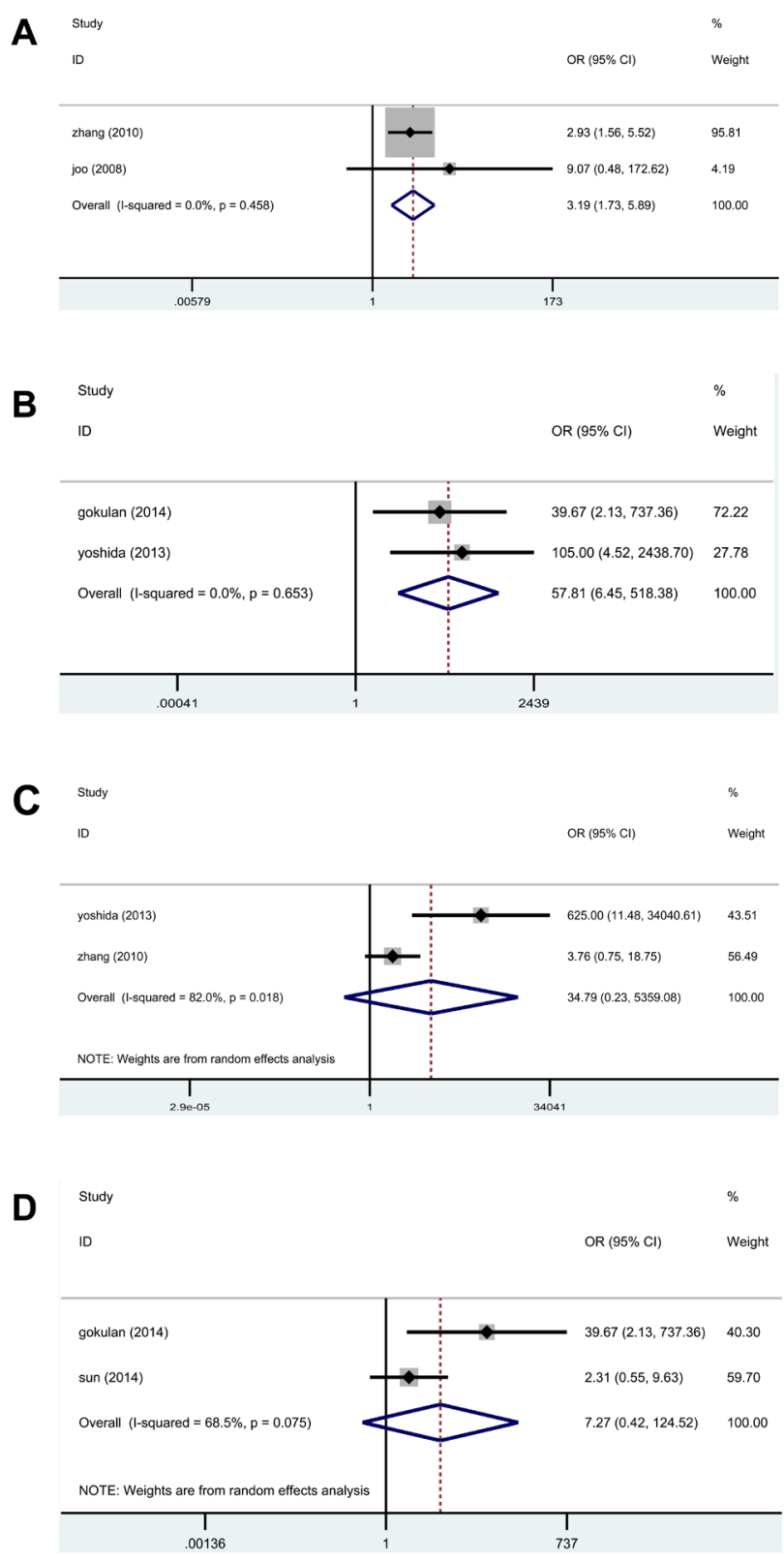

\section{The role of prognosis of Notch signaling pathway}

Four studies reported the prognostic of the Notch signaling pathway. Two studies focused on Notch1. Meta-analysis results revealed that over-expression of Notch1 was associated with poor prognosis $(\mathrm{HR}=0.74$, 95\%CI: 0.12-1.35; a fixed effects analysis, $\mathrm{p}=0.019)$ (Fig. 4). The Kaplan-Meier and log-rank analysis revealed that a high expression of NICD and HES1 was inclined to poor survival. ${ }^{17}$ Zhang et al. reported that elevated DLL4 expression independently predicts poor prognosis. ${ }^{19}$

\section{Publication bias}

Publication bias analysis was performed among studies based on Notch1 expression. The funnel plots were asymmetric (Figure not shown). This may be due to the number of eligible studies.

\section{Discussion}

Notch phenotypes were first identified 100 years ago in Drosophila melanogaster. Accumulating evidence indicates that Notch signaling plays a vital role in cancer stem cell, cell fate determination and differentiation and tumor angiogenesis. ${ }^{25}$ However, whether Notch signaling acts as an oncogene or a tumor suppressor is still controversial. In lung cancer, the activation of Notch signaling promotes hypoxia induced epithelial mesenchymal transition. ${ }^{26}$ In melanoma, activated Notch signaling enhances the adhesion and metastasis of melanoma cell by up-regulating $\mathrm{N}$-cadherin. ${ }^{27} \mathrm{On}$ the contrary, inhibition of Notch signaling can promote skin squamous cell carcinoma formation. ${ }^{28}$ Similarly, the role of Notch signaling is controversial in HNSCC. In this meta-analysis, we investigated the association between Notch signaling pathway and HNSCC. We found that the expression of Notch1, Notch3 and NICD was increased in HNSCC as compared with control tissue. And there was no statistically significant difference in Jagged1 and HES1 expression between HNSCC and control tissue. Further, we searched the relationship between Notch signaling and prognosis of HNSCC patients. Notch1 can be used as an independent predict biomarker in HNSCC patients.

In the current meta-analysis, we retrieved 5 studies focused on Notch1 expression in HNSCC and 2 studies showed the correlation between Notch1 expression and prognosis in HNSCC patients. According to the results of the meta-analysis, Notch 1 expression was significantly up-regulated in HNSCC. However, Notch1 mutations were detected in approximate 15\% of HNSCC. Moreover, Notch1 mutations were loss-of-function mutation and played a tumor suppressive role in HNSCC development. ${ }^{16}$ Sakamoto et al. also reported that the expression of Notch1 was decreased in OSCC as compared with control tissue. ${ }^{29}$ Although, there was a litter subset of HNSCC exhibiting loss-of-function mutations, the activation of Notch1 is found in major HNSCC. This is consistent with our results of this meta-analysis. Additionally, Notch1 was found to be more preferably expressed in HNSCC patients who had lymph node metastasis and poor clinical stage. This suggested that the activation of Notch1 might promote epithelial-mesenchymal transition and sustain the cancer stem cell.

Irregular Notch3 activity has been associated with some solid tumors, such as breast cancer and lung cancer. ${ }^{30,31}$ Studies revealed that Notch3 expression is lim- 
Fig. 4. Meta analysis of Notch1 expression and survival of HNSCC patients. Forest plot of RR for overall survival

Study

ID

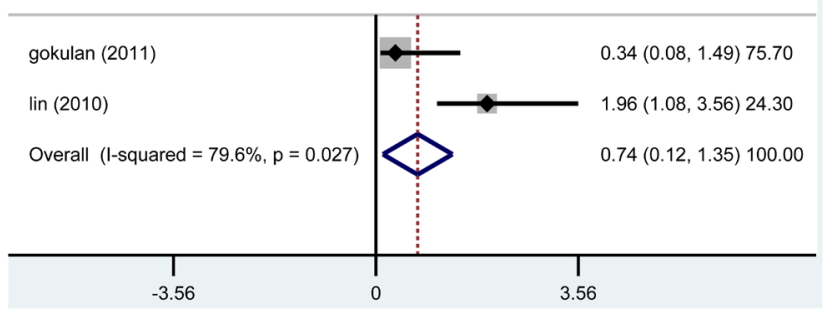

ited to the mammal's vascular system. In a mouse model, knockout Notch3 results in vascular smooth vessel defects, suggesting a relationship of Notch3 with angiogenesis. ${ }^{32}$ Given the role of Notch3 in angiogenesis, the activation of Notch3 probably promotes tumor development by increasing angiogenesis in cancer. In this metaanalysis, we found that Notch3 had a significantly high expression in HNSCC as compared with control tissue. Notch3 expression also had a significant correlation with clinical stage in HNSCC patients.

The Notch1 ligand Jagged1 was demonstrated to be involved in tumor progress. ${ }^{33}$ Blockage of Jagged1-mediated Notch signaling reduced tumor growth by decreasing angiogenesis. ${ }^{34}$ In this meta-analysis, we found that the expression of Jagged1 was up-regulated in HNSCC, especially in positive lymph node metastasis patients. Besides, Jagged1 inhibition of Notch1 ligand Jagged2 similarly decreased tumor growth by reducing angiogenesis. ${ }^{34}$ However, Zhang et al. reported that the expression of Jagged2 did not have a statistically significant difference in HNSCC tissue and control tissue. NICD acting as Notch intracellular fragment was involved in tumor cell proliferation, apoptosis and angiogenesis. ${ }^{35}$ The activation of NICD translating into the nucleus can regulate the expression of target genes, such as HES1 and HEY1. ${ }^{5}$ Mechanism research showed that HES1 plays a key role in stemness, metastasis and multi-drug resistance. ${ }^{36}$ The meta-analysis revealed that NICD had a higher expression in HNSCC than in control tissue. As only 2 studies focused on HES1 expression, there was no statistically significant difference in HES1 expression between HNSCC and control tissue. The data from the Cancer Genome Atlas (TGGA) database suggested that the expression of HES1 is enhanced in HNSCC. ${ }^{16}$ Ravindran reported that both NICD and HES1 were correlated with prognosis in HNSCC. ${ }^{17}$ This data indicated that the Notch signaling pathway plays a critical role HNSCC development.

There are several limitations in current meta-analysis. Firstly, the number of eligible studies and the number of studies including HNSCC patients are too scarce. Secondly, there are differences in the criteria for Notch signaling evaluation. Although IHC method was applied in all included studies, the cutoff values of positive ranges from 5 to $15 \%$. Thirdly, the funnel plot analysis showed publication bias and is still a concern in these studies.

\section{Conclusion}

Taken together, our meta-analysis indicated that the Notch signaling pathway was activated in HNSCC. The Notch signaling pathway was associated with partial clinicopathological characteristics of HNSCC, such as clinical stage, lymph node metastasis status and differentiation. Notch signaling may be used as a poor prognostic biomarker for HNSCC. Inhibition of Notch signaling pathway is a potential therapeutic method of HNSCC. Since the number of studies is relatively limited, further research should be performed to investigate the precise role of the Notch signaling pathway in HNSCC.

\section{References}

1. Torre LA, Bray F, Siegel RL, et al. Global cancer statistics, 2012. CA Cancer J Clin. 2015;65:87-108.

2. Tien AC, Rajan A, Bellen HJ. A Notch updated. J Cell Biol. 2009;184:621-629.

3. Ranganathan $\mathrm{P}$, Weaver $\mathrm{KL}$, Capobianco AJ. Notch signalling in solid tumours: A little bit of everything but not all the time. Nat Rev Cancer. 2011;11:338-351.

4. Sethi N, Dai X, Winter CG, Kang Y. Tumor-derived JAGGED1 promotes osteolytic bone metastasis of breast cancer by engaging notch signaling in bone cells. Cancer Cell. 2001;19:192-205.

5. Kalaitzidis D, Armstrong SA. Cancer: The flipside of Notch. Nature. 2001;473:159-160.

6. Hori K, Sen A, Artavanis-Tsakonas S. Notch signaling at a glance. J Cell Sci. 2013;126:2135-2140.

7. Pece S, Serresi M, Santolini E, et al. Loss of negative regulation by Numb over Notch is relevant to human breast carcinogenesis. J Cell Biol. 2004;167:215-221.

8. Grabher $\mathrm{C}$, von Boehmer $\mathrm{H}$, Look AT. Notch 1 activation in the molecular pathogenesis of T-cell acute lymphoblastic leukaemia. Nat Rev Cancer. 2006;6:347-359.

9. Lin $\mathrm{CL}$, Wang FS, Hsu YC, et al. Modulation of notch-1 signaling alleviates vascular endothelial growth factor-mediated diabetic nephropathy. Diabetes. 2010;59:1915-1925.

10. Tao J, Jiang MM, Jiang $L$, et al. Notch activation as a driver of osteogenic sarcoma. Cancer Cell. 2014;26:390-401.

11. Nicolas M, Wolfer A, Raj K, et al. Notch1 functions as a tumor suppressor in mouse skin. Nat Genet. 2003;33:416-421.

12. Wang WM, Zhao ZL, Ma SR, et al. Epidermal growth factor receptor inhibition reduces angiogenesis via hypoxia-inducible factor1alpha and Notch1 in head neck squamous cell carcinoma. PLoS One. 2015;10:e0119723.

13. Shrivastava S, Steele R, Sowadski M, et al. Identification of molecular signature of head and neck cancer stem-like cells. Sci Rep. 2015;5:7819.

14. Gu F, Ma Y, Zhang Z, et al. Expression of Stat3 and Notch1 is associated with cisplatin resistance in head and neck squamous cell carcinoma. Oncol Rep. 2010;23:671-676.

15. Agrawal N, Frederick MJ, Pickering CR, et al. Exome sequencing of head and neck squamous cell carcinoma reveals inactivating mutations in NOTCH1. Science. 2011;333:1154-1157.

16. Sun W, Gaykalova DA, Ochs MF, et al. Activation of the NOTCH pathway in head and neck cancer. Cancer Res. 2014;74:1091-1104.

17. Gokulan R, Halagowder D. Expression pattern of Notch intracellular domain (NICD) and Hes-1 in preneoplastic and neoplastic human oral squamous epithelium: Their correlation with c-Myc, clinicopathological factors and prognosis in Oral cancer. Med Oncol. 2014;31:126. 
18. Yoshida R, Nagata M, Nakayama $H$, et al. The pathological significance of Notch1 in oral squamous cell carcinoma. Lab Invest. 2013;93:1068-1081.

19. Zhang JX, Cai MB, Wang XP, et al. Elevated DLL4 expression is correlated with VEGF and predicts poor prognosis of nasopharyngeal carcinoma. Med Oncol. 2013;30:390.

20. Ravindran G, Devaraj H. Aberrant expression of beta-catenin and its association with DeltaNp63, Notch-1, and clinicopathological factors in oral squamous cell carcinoma. Clin Oral Investig. 2012;16:1275-1288.

21. Zhang TH, Liu HC, Zhu LJ, et al. Activation of Notch signaling in human tongue carcinoma. J Oral Pathol Med. 2011;40:37-45.

22. Lin JT, Chen MK, Yeh KT, et al. Association of high levels of Jagged-1 and Notch-1 expression with poor prognosis in head and neck cancer. Ann Surg Oncol. 2010;17:2976-2983.

23. Joo YH, Jung CK, Kim MS, Sun DI. Relationship between vascular endothelial growth factor and Notch1 expression and lymphatic metastasis in tongue cancer. Otolaryngol Head Neck Surg. 2009;140:512-518.

24. Zhang ZP, Sun YL, Fu L, et al. Correlation of Notch1 expression and activation to cisplatin-sensitivity of head and neck squamous cell carcinoma. Ai Zheng. 2009;28:100-103.

25. Ntziachristos P, Lim JS, Sage J, Aifantis I. From fly wings to targeted cancer therapies: A centennial for notch signaling. Cancer Cell. 2014;25:318-334.

26. Gao XJ, Liu JW, Zhang QG, et al. Nobiletin inhibited hypoxiainduced epithelial-mesenchymal transition of lung cancer cells by inactivating of Notch-1 signaling and switching on miR-200b. Pharmazie. 2015;70:256-262.

27. Zhang JP, Li N, Bai WZ, et al. Notch ligand Delta-like 1 promotes the metastasis of melanoma by enhancing tumor adhesion. Braz J Med Biol Res. 2014;47:299-306.

28. Proweller A, Tu L, Lepore JJ, et al. Impaired notch signaling promotes de novo squamous cell carcinoma formation. Cancer Res. 2006;66:7438-7444.

29. Sakamoto K, Fujii T, Kawachi H, et al. Reduction of NOTCH1 expres sion pertains to maturation abnormalities of keratinocytes in squamous neoplasms. Lab Invest. 2012;92:688-702.

30. Yamaguchi $\mathrm{N}$, Oyama $\mathrm{T}$, Ito $\mathrm{E}$, et al. NOTCH3 signaling pathway plays crucial roles in the proliferation of ErbB2-negative human breast cancer cells. Cancer Res. 2008;68:1881-1888.

31. Lin L, Mernaugh R, Yi F, et al. Targeting specific regions of the Notch3 ligand-binding domain induces apoptosis and inhibits tumor growth in lung cancer. Cancer Res. 2010;70:632-638.

32. Domenga $V$, Fardoux $P$, Lacombe $P$, et al. Notch3 is required for arterial identity and maturation of vascular smooth muscle cells. Genes Dev. 2004;18:2730-2735.

33. Xu Y, Zhu F, Xu S, Liu L. Anti-tumor effect of the extract from qingyihuaji formula on pancreatic cancer by down-regulating Notch4 and Jagged-1. J Tradit Chin Med. 2015;35:77-83.

34. Kangsamaksin T, Murtomaki A, Kofler NM, et al. NOTCH decoys that selectively block DLL/NOTCH or JAG/NOTCH disrupt angiogenesis by unique mechanisms to inhibit tumor growth. Cancer Discov. 2015;5:182-197.

35. Gopalakrishnan N, Saravanakumar M, Madankumar P, Thiyagu M, Devaraj $\mathrm{H}$. Colocalization of beta-catenin with Notch intracellular domain in colon cancer: A possible role of Notch1 signaling in activation of CyclinD1-mediated cell proliferation. Mol Cell Biochem. 2014;396:281-293.

36. Liu ZH, Dai XM, Du B. Hes1: A key role in stemness, metastasis and multidrug resistance. Cancer Biol Ther. 2015;16:353-359. 